\title{
Nomogram for the prediction of postoperative hypoxemia in patients with acute aortic dissection
}

\author{
Huiqing Ge', Ye Jiang ${ }^{1}$, Qijun Jin ${ }^{1}$, Linjun Wan ${ }^{1}$, Ximing Qian² and Zhongheng Zhang ${ }^{3^{*}}$ (D)
}

\begin{abstract}
Background: Postoperative hypoxemia is quite common in patients with acute aortic dissection (AAD) and is associated with poor clinical outcomes. However, there is no method to predict this potentially life-threatening complication. The study aimed to develop a regression model in patients with AAD to predict postoperative hypoxemia, and to validate it in an independent dataset.

Methods: All patients diagnosed with AAD from December 2012 to December 2017 were retrospectively screened for potential eligibility. Preoperative and intraoperative variables were included for analysis. Logistic regression model was fit by using purposeful selection procedure. The original dataset was split into training and validating datasets by 4:1 ratio. Discrimination and calibration of the model was assessed in the validating dataset. A nomogram was drawn for clinical utility.

Results: A total of 211 patients, involving 168 in non-hypoxemia and 43 in hypoxemia group, were included during the study period (incidence: $20.4 \%$ ). Duration of mechanical ventilation (MV) was significantly longer in the hypoxemia than non-hypoxemia group $(41(10.5140)$ vs. $12(3.75,70.25)$ hours; $p=0.002)$. There was no difference in the hospital mortality rate between the two groups. The purposeful selection procedure identified 8 variables including hematocrit (odds ratio [OR]: $0.89,95 \%$ confidence interval [Cl]: 0.80 to $0.98, p=0.011), \mathrm{PaO}_{2} / \mathrm{FiO}_{2}$ ratio (OR: $0.99,95 \% \mathrm{Cl}$ : 0.99 to $1.00, p=0.011$ ), white blood cell count (OR: $1.21,95 \% \mathrm{Cl}: 1.06$ to $1.40, p=0.008$ ), body mass index (OR: 1.32, 95\% Cl: 1.15 to $1.54 ; p=0.000)$, Stanford type (OR: $0.22,95 \%$ Cl: 0.06 to $0.66 ; p=0.011), \mathrm{pH}$ (OR: $0.0002,95 \%$ Cl: $2^{*} 10^{-8}$ to 0. 74; $p=0.048$ ), cardiopulmonary bypass time (OR: $0.99,95 \%$ Cl: 0.98 to $1.00 ; p=0.031$ ) and age (OR: $1.03,95 \%$ Cl: 0.99 to $1.08 ; p=0.128$ ) to be included in the model. In an independent dataset, the area under curve (AUC) of the prediction model was 0.869 ( $95 \%$ Cl: 0.802 to 0.936 ). The calibration was good by visual inspection.
\end{abstract}

Conclusions: The study developed a model for the prediction of postoperative hypoxemia in patients undergoing operation for AAD. The model showed good discrimination and calibration in an independent dataset that was not used for model training.

Keywords: Acute aortic dissection, Hypoxemia, Nomogram, Intensive care unit, Length of stay

\footnotetext{
* Correspondence: zh_zhang1984@zju.edu.cn

${ }^{3}$ Department of Emergency Medicine, Sir Run Run Shaw Hospital, Zhejiang

University School of Medicine, No 3, East Qingchun Road, Hangzhou 310016,

Zhejiang Province, China

Full list of author information is available at the end of the article
}

(c) The Author(s). 2018 Open Access This article is distributed under the terms of the Creative Commons Attribution 4.0 International License (http://creativecommons.org/licenses/by/4.0/), which permits unrestricted use, distribution, and reproduction in any medium, provided you give appropriate credit to the original author(s) and the source, provide a link to the Creative Commons license, and indicate if changes were made. The Creative Commons Public Domain Dedication waiver (http://creativecommons.org/publicdomain/zero/1.0/) applies to the data made available in this article, unless otherwise stated. 


\section{Background}

Acute aortic dissection (AAD) represents a life-threatening condition that can be encountered in emergency and critical care setting [1]. Many factors can influence the clinical outcomes of these patients such as the comorbidities, complications, organ dysfunction and site of dissection. Surgical operation is usually needed to avert catastrophic complications of aortic dissection [2]. Postoperative hypoxemia has long been noted in substantial proportion of patients with $\mathrm{AAD}$ and has been found to be associated with poor clinical outcomes such as prolonged mechanical ventilation, increased length of stay (LOS) in the intensive care unit (ICU) and hospital [3-5]. Also, several studies have attempted to identify preoperative risk factor of hypoxemia $[4,5]$. However, there is no report on training a model for early prediction of postoperative hypoxemia. Since early prediction of post-operative hypoxemia makes early intervention possible, it is of clinical utility to train and validate such a prediction model. The study aimed to develop a model for early prediction of postoperative hypoxemia. Discrimination and calibration of the model were validated in an independent dataset that was not used for model training. A nomogram was depicted for clinical use.

\section{Methods}

\section{Study design and settings}

The study was retrospective in design. All patients diagnosed as AAD from December 2012 to December 2017 were screened for potential eligibility. The patients were identified from the electronic healthcare record (EHR) of our hospital. Patients with initial suspected diagnosis of AAD as denoted by ICD9 code of 443.21 were identified. Exclusion criteria included one of the following items: 1) patients did not undergo surgery; 2) patients who were pregnant, or had neuromuscular disease, 3) confirmed complications such as heart failure, massive bleeding, pneumothorax, tracheal hemorrhage, atelectasis and pneumonia; and 3) patients had missing values on more than $50 \%$ variables. Data were extracted from EHR and deidentified before analysis. The study was approved by the ethics committee of Sir Run Run Shaw hospital (20180611-7). Informed consent was waived due to retrospective nature of the study.

\section{Variables included for analysis}

Demographic variables including age, gender, body weight, height, body mass index (BMI) were obtained for the hospital admission with surgical repair of the aorta artery. Past histories of smoking, hypertension and diabetes mellitus were also included. The admission type included emergency and non-emergency admissions.

Preoperative laboratory tests were obtained within $24 \mathrm{~h}$ before surgery, which included albumin, hematocrit
(HCT), $\mathrm{pH}$, lactate, $\mathrm{PaCO} 2, \mathrm{PaO} 2 / \mathrm{FiO} 2$ ratio $(\mathrm{P} / \mathrm{F})$, serum creatinine $(\mathrm{Scr})$, total bilirubin, white blood cell count (WBC), C-reactive protein (CRP), troponin, creatine kinase $(\mathrm{CK})$, creatine kinase isoenzymes (CKMB), lactate dehydrogenase (LDH), aspartate aminotransferase (AST). If there were two or more measures of these variables before operation, the one nearest to the operation was employed.

Intraoperative variables included aortic clamping time, cardiopulmonary bypass $(\mathrm{CPB})$ time, duration of the operation, fluid input and output during operation, and the minimum body temperature.

Clinical outcomes were LOS in ICU and hospital, duration of postoperative mechanical ventilation, and hospital mortality.

Postoperative hypoxemia was defined as $\mathrm{P} / \mathrm{F}<200$ for the first 2 days after operation.

\section{Statistical analysis}

Continuous variables were expressed as mean and standard deviation for normally distributed data, and as median and interquartile range (IQR) for non-normal data. Categorical variables were expressed as number and percentage. Comparisons between hypoxemia and non-hypoxemia groups were performed using student $\mathrm{t}$ test or rank sum test as appropriate. Chi-square or Fisher's exact test was employed for categorical variables $[6,7]$.

Postoperative hypoxemia was employed as response variable assuming a binomial distribution, and covariates were included in the model if their $p$ values were less than 0.05 in univariate analysis [8]. Other variables such as age, $\mathrm{CPB}$ time and $\mathrm{pH}$ were entered due to clinical expertise. Variables with $p>0.2$ in the multivariable model were excluded. The initial dataset was randomly split into the training and validating dataset by $4: 1$ ratio (there were 36 cases of hypoxemia in the training set). The training set was used to develop the model, and the validating set was used to validate the model. Model discrimination was represented by the area under receiver operating characteristic curves (AUC), with an AUC greater than 0.8 indicating a good discrimination [9]. Model calibration was visually assessed by plotting fitted logistic calibration curve and a smooth nonparametric fit using lowess and grouped proportions vs. mean predicted probability in group [10]. Other statistics were also reported with the R package $R e$ gression Modeling Strategies (rms), such as Somers'D_\{xy\}, rank correlation between predicted probability and observed probability, Nagelkerke-Cox-Snell-Maddala-Magee R-squared index, the 0.9 quantile of same (E90), the Spiegelhalter Z-test for calibration accuracy, and its two-tailed $P$-value $[11,12]$.

A nomogram was drawn based on the fitted logistic regression model and each patient could be mapped 
onto the nomogram for the prediction of the occurrence of postoperative hypoxemia [13]. We drew the nomogram that converts each effect in the model to a 0 to 100 scale which is just proportional to the log odds. These points are added across predictors to derive the "Total Points," which are converted to linear predictor and then to predicted probabilities [12]. The distribution of covariates in the model, and of the total regression score, are superimposed on the nomogram scales. Also, the values of a sample patient were superimposed. Binomial distribution with logit link function was employed for the model fit.

All statistical analyses were performed using $\mathrm{R}$ (version 3.4.3). Two-tailed $p$ value less than 0.05 was considered as statistical significance.

\section{Results}

\section{Patient inclusion}

A total of 287 patients with initial suspicion of AAD were initially identified from HER, and 49 were excluded because they did not undergo surgery during hospital stay, were ruled out for AAD, had neuromuscular disease. Furthermore, we excluded 27 patients with preoperative complications such as heart failure, massive bleeding, pneumothorax, atelectasis and pneumonia. As a result, a total of 211 patients were finally included for analysis (Fig. 1).

\section{Baseline characteristics}

There were 168 patients in the non-hypoxemia group and 43 in the hypoxemia group, with an incidence rate

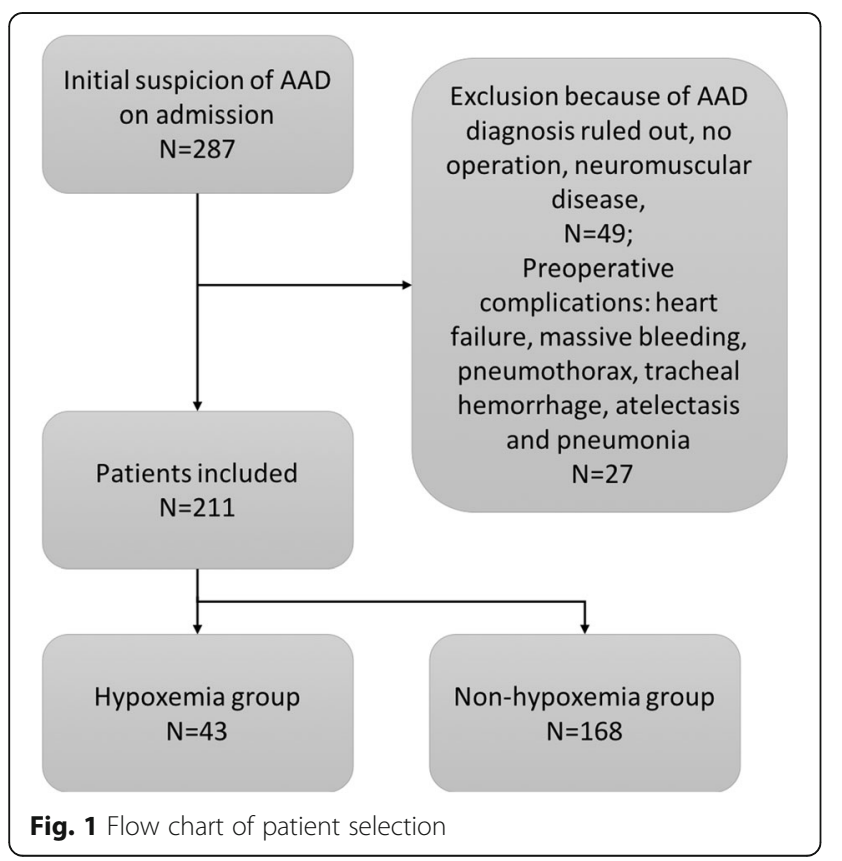

of $20.4 \%$. Patients in the hypoxemia group appears to be elder than non-hypoxemia group, but the statistical significance was not reached (Table 1). Patients with hypoxemia showed significantly greater BMI than those in non-hypoxemia group $(26.82 \pm 3.84$ vs. $24.94 \pm$ $3.86 \mathrm{~kg} / \mathrm{m} 2, p=0.006)$. All patients $(100 \%)$ with hypoxemia were admitted from emergency setting, versus $86 \%$ for the non-hypoxemia group $(p=0.005)$.

\section{Preoperative laboratory test}

For preoperative laboratory tests, hypoxemia patients showed significantly lower $\mathrm{P} / \mathrm{F}$ ratio than non-hypoxemia patients (median, interquartile range [IQR]: 174(148.5237) vs. $249(183.75,367.25) ; p<0.001)$. Other laboratory tests such as serum creatinine $(103(77.5145)$ vs. $76.5(61,104)$ $\mathrm{mmol} / \mathrm{l} ; \quad p<0.001)$, total bilirubin $(16.7(11.9,25)$ vs. 13.2(9.57,18.9) $\mathrm{mmol} / \mathrm{l} ; p=0.016), \operatorname{WBC}(12.9(9.3,15.5)$ vs. $\left.8.9(6.8,11.65) \wedge 10^{9} / 1 ; p<0.001\right)$, CRP $(58.9(10.6118 .3)$ vs. $23.4(4.57,60.38) \mathrm{mg} / \mathrm{l} ; p=0.003)$ and CK $(109(62,228)$ vs. $72(48.75,136.25) \mathrm{U} / \mathrm{l} ; p=0.023)$ were significantly higher in the hypoxemia group than that in the non-hypoxemia group.

Intraoperative variables were not significantly different between hypoxemia and non-hypoxemia groups.

\section{Clinical outcomes}

The LOS in ICU $(10(5.5,14)$ vs. $7(4,11)$ days; $p=0.079)$ and hospital $(18(12.5,24)$ vs. $19(13,25)$ days; $p=0.775)$ were not significantly different between the two groups. Duration of MV was significantly longer in the hypoxemia than non-hypoxemia group $(41(10.5140)$ vs. $12(3.75,70.25)$ hours; $p=0.002)$. There was no difference in the hospital mortality rate between the two groups (Table 1).

\section{Model training}

Logistic regression model was fit with the training set. The purposeful selection procedure included 8 variables as determined by clinical importance and statistical significance (Table 2). The preoperative HCT (odds ratio [OR]: $0.89,95 \%$ confidence interval [CI]: 0.80 to $0.98, p$ $=0.011$ ), P/F ratio (OR: $0.99,95 \%$ CI: 0.99 to $1.00, p=$ 0.011 ), WBC (OR: $1.21,95 \%$ CI: 1.06 to $1.40, p=0.008$ ), BMI (OR: $1.32,95 \%$ CI: 1.15 to $1.54 ; p<0.001$ ), Stanford type (OR: $0.22,95 \% \mathrm{CI}: 0.06$ to $0.66 ; p=0.011$ ), $\mathrm{pH}$ (OR: $0.0002,95 \% \mathrm{CI}: 2^{*} 10^{-8}$ to $0.74 ; p=0.048$ ), and CPB time (OR: $0.99,95 \%$ CI: 0.98 to $1.00 ; p=0.031$ ) were significantly contributing to the prediction accuracy of the model. Age was not significantly associated with the outcome, but it remained in the model by clinical judgement. 
Table 1 Comparison between hypoxemia and non-hypoxemia groups

\begin{tabular}{|c|c|c|c|c|}
\hline & Total $(n=211)$ & Non-hypoxemia $(n=168)$ & Hypoxemia $(n=43)$ & $\mathrm{p}$ \\
\hline \multicolumn{5}{|l|}{ Demographics } \\
\hline Age, median (IQR) (years) & $50(43,63)$ & $50(42,64)$ & $52(46.5,59)$ & 0.654 \\
\hline Male, n (\%) & $151(0.72)$ & $116(0.69)$ & $35(0.81)$ & 0.158 \\
\hline Height, median (IQR) (cm) & $170(163,174)$ & $170(162.75,174)$ & $169(165,174)$ & 0.647 \\
\hline Weight, median (IQR) (kg) & $70(62,80)$ & $70(60,80)$ & $80(65,89.5)$ & 0.005 \\
\hline BMl, mean \pm SD (kg/m2) & $25.32 \pm 3.92$ & $24.94 \pm 3.86$ & $26.82 \pm 3.84$ & 0.006 \\
\hline Smoking history, n (\%) & $59(28)$ & $47(28)$ & $12(28)$ & 0.998 \\
\hline Hypertension, n (\%) & $134(64)$ & $102(61)$ & $32(74)$ & 0.137 \\
\hline Diabetes, n (\%) & $13(6)$ & $11(7)$ & $2(5)$ & 0.998 \\
\hline Stanford A, n (\%) & $133(63)$ & $97(0.58)$ & $36(84)$ & 0.003 \\
\hline Emergency admission, n (\%) & $188(89)$ & $145(86)$ & $43(1)$ & 0.005 \\
\hline \multicolumn{5}{|l|}{ Laboratory tests before operation } \\
\hline Albumin, mean $\pm \mathrm{SD}(\mathrm{mg} / \mathrm{l})$ & $36.6 \pm 4.65$ & $36.75 \pm 4.76$ & $36 \pm 4.22$ & 0.316 \\
\hline Hematocrit, mean \pm SD (\%) & $36.09 \pm 5.71$ & $36.26 \pm 5.4$ & $35.41 \pm 6.82$ & 0.452 \\
\hline pH, median (IQR) & $7.37(7.34,7.4)$ & $7.37(7.34,7.4)$ & $7.37(7.32,7.38)$ & 0.205 \\
\hline Lactate, median (IQR) (mmol/l) & $2.1(1.5,2.9)$ & $2.05(1.48,2.8)$ & $2.30(1.9,3.1)$ & 0.082 \\
\hline $\mathrm{PaCO} 2$, median (IQR) (mmHg) & $41(37.55,45.55)$ & $41(37.88,45.23)$ & $40.9(36.5,47.7)$ & 0.916 \\
\hline $\mathrm{P} / \mathrm{F}$ ratio, median (IQR) & $235(176,355)$ & $249(183.75,367.25)$ & $174(148.5237)$ & $<0.001$ \\
\hline Scr, median (IQR) (mmol/l) & $81(64,113)$ & $76.5(61,104)$ & $103(77.5145)$ & $<0.001$ \\
\hline Total bilirubin, median (IQR) (mmol/l) & $13.7(10,22.25)$ & $13.2(9.57,18.9)$ & $16.7(11.9,25)$ & 0.016 \\
\hline WBC, median $(\mathrm{IQR})(\wedge 109 / \mathrm{I})$ & $9.5(7.4,12.6)$ & $8.9(6.8,11.65)$ & $12.9(9.3,15.5)$ & $<0.001$ \\
\hline CRP, median (IQR) (mg/l) & $28.3(5.75,68.75)$ & $23.4(4.57,60.38)$ & $58.9(10.6118 .3)$ & 0.003 \\
\hline Troponin, median (IQR) (ng/ml) & $0.01(0.01,0.01)$ & $0.01(0.01,0.01)$ & $0.01(0.01,0.12)$ & 0.028 \\
\hline CK, median (IQR) (U/I) & $78(51,150.5)$ & $72(48.75,136.25)$ & $109(62,228)$ & 0.023 \\
\hline CKMB, median (IQR) (U/I) & $10(8,16)$ & $10(8,16)$ & $10(6.5,14.5)$ & 0.249 \\
\hline LDH, median (IQR) $(\mathrm{U} / \mathrm{I})$ & $218(171,283)$ & $208.5(165,274.25)$ & $253(196.5312 .5)$ & 0.013 \\
\hline AST, median (IQR) (U/l) & $22(16.5,34.5)$ & $21(15,31.25)$ & $31(18.5,44.5)$ & 0.007 \\
\hline \multicolumn{5}{|l|}{ Intraoperative variables } \\
\hline Aortic clamping time, mean \pm SD (min) & $127.91 \pm 40.82$ & $128.84 \pm 40.7$ & $124.26 \pm 41.59$ & 0.519 \\
\hline CBP time, median (IQR) (min) & $178(140,211)$ & $180(146.75,210)$ & $169(132.5221)$ & 0.749 \\
\hline Duration of operation, median (IQR) min & $295(120,390)$ & $285(90,390)$ & $300(242.5420)$ & 0.072 \\
\hline Minimum temperature, median $(\mathrm{IQR})\left({ }^{\circ} \mathrm{C}\right)$ & $36.2(35.6,36.55)$ & $36.2(35.4,36.6)$ & $36.3(36,36.5)$ & 0.250 \\
\hline Input, median (IQR) (ml) & $6250(5150,7587.5)$ & $6090(5150,7332.5)$ & $6380(4775,8170)$ & 0.651 \\
\hline Output, median (IQR) (ml) & $4850(3600,5800)$ & $4900(3575,6000)$ & $4500(3675,5600)$ & 0.358 \\
\hline \multicolumn{5}{|l|}{ Clinical outcomes } \\
\hline LOS in ICU, median (IQR) (days) & $7(4,12)$ & $7(4,11)$ & $10(5.5,14)$ & 0.079 \\
\hline LOS in hospital, median (IQR) (days) & $19(13,24.5)$ & $19(13,25)$ & $18(12.5,24)$ & 0.775 \\
\hline Duration of MV, median (IQR) (hours) & $15(5,82)$ & $12(3.75,70.25)$ & $41(10.5140)$ & 0.002 \\
\hline Mortality, n (\%) & $7(3)$ & $6(4)$ & $1(2)$ & 0.997 \\
\hline
\end{tabular}

Note: continuous variables were expressed as mean and standard deviation for normal data, and as median and interquartile range for non-normal data. Categorical variables were expressed as number and percentage. Comparisons between hypoxemia and non-hypoxemia groups were performed using student $\mathrm{t}$ test or rank sum test as appropriate. Chi-square or Fisher's exact test was employed for categorical variables

Abbreviations: No. number, Prop. proportion, ICU intensive care unit, LOS length of stay, WBC white blood cell count, CRP c-reactive protein, Scr serum creatinine, $B M I$ body mass index, $C K$ creatine kinase, $C K M B$ creatine kinase isoenzymes, $L D H$ lactate dehydrogenase, $A S T$ aspartate aminotransferase, $M V$ mechanical ventilation, $C P B$ cardiopulmonary bypass, IQR interquartile range, $S D$ standard deviation 
Table 2 Logistic regression model for the prediction of postoperative hypoxemia

\begin{tabular}{lllll}
\hline Variables & Odds ratio & Lower limit of 95\% Cl & Upper limit of 95\% Cl & $P$ value \\
\hline BMI & 1.32 & 1.15 & 1.54 & 1.00 \\
PF & 0.99 & 0.99 & 0.001 \\
Stanford (A as reference) & 0.22 & 0.06 & 1.40 & 0.011 \\
WBC & 1.21 & 1.06 & 1.08 & 0.011 \\
Age & 1.03 & 0.99 & 0.98 & 0.008 \\
HCT & 0.89 & 0.80 & 1.00 & 0.128 \\
CBP time & 0.99 & 0.98 & 0.74 & 0.016 \\
PH & 0.0002 & $2 * 10^{-8}$ & 0.048
\end{tabular}

Note: The logistic regression model was selected by using stepwise forward selection procedure, AIC was used to decide the inclusion of a variable. The odds ratio was reported for each one unit increase for each variable

Abbreviations: $\mathrm{Cl}$ confidence interval, WBC white blood cell count, PF PaO2/FiO2, BMI body mass index, CBP cardiopulmonary bypass, HCT hematocrit

\section{Model validation in a separate dataset}

In the validation dataset, the fitted model showed a good discrimination in distinguishing hypoxemia from non-hypoxemia patients (area under curve [AUC] = 0.869, 95\% CI: 0.802 to 0.936 ). Model calibration was shown in Fig. 2. The non-parametric curve fits well to the ideal line, indicating the observed probability was in line with the predicted probability. However, the model may not predict well for patients with lower risk of postoperative hypoxemia. Also, the Fig. 3 shows that the predicted probability of hypoxemia is in agreement with the observed proportion.

\section{Nomogram for predicting postoperative hypoxemia}

Nomogram for the prediction of postoperative hypoxemia is shown in Fig. 4. The distribution of each variable was shown above each line. A representative patient was shown to illustrate how to use the nomogram. Given values of the 8 predictors, the patient can be mapped to the nomogram. Note there is a red dot in each line, representing the value of each of the 8 predictors for the patient. Regression coefficient of each predictor was scaled to points within the range of 0 to 100 , and the relative importance (weight) of each predictor can be reflected by its

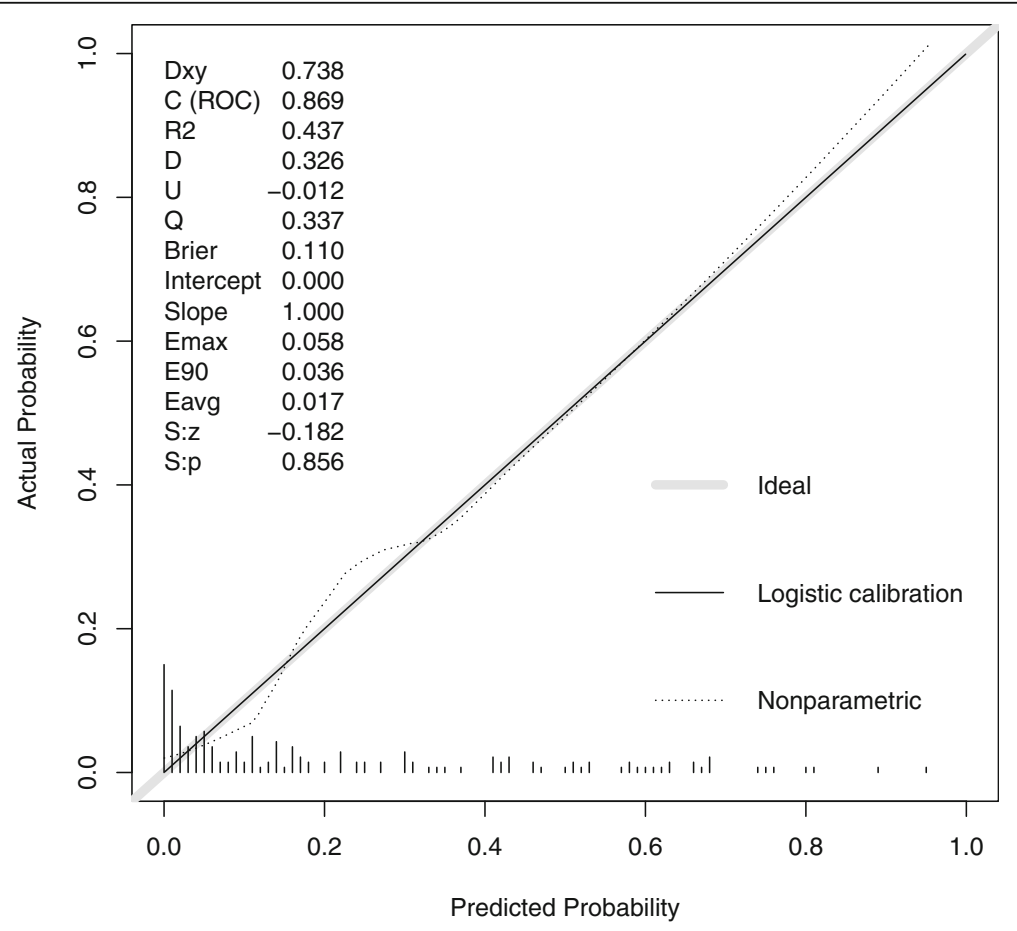

Fig. 2 Model calibration and discrimination. The predicted probability by the logistic regression model conforms well to the observed probability well. In the plot, the idea line is consistent with the Logistic calibration line. The discrimination was measured by AUC of 0.869 (95\% Cl: 0.802 to 0.936) 


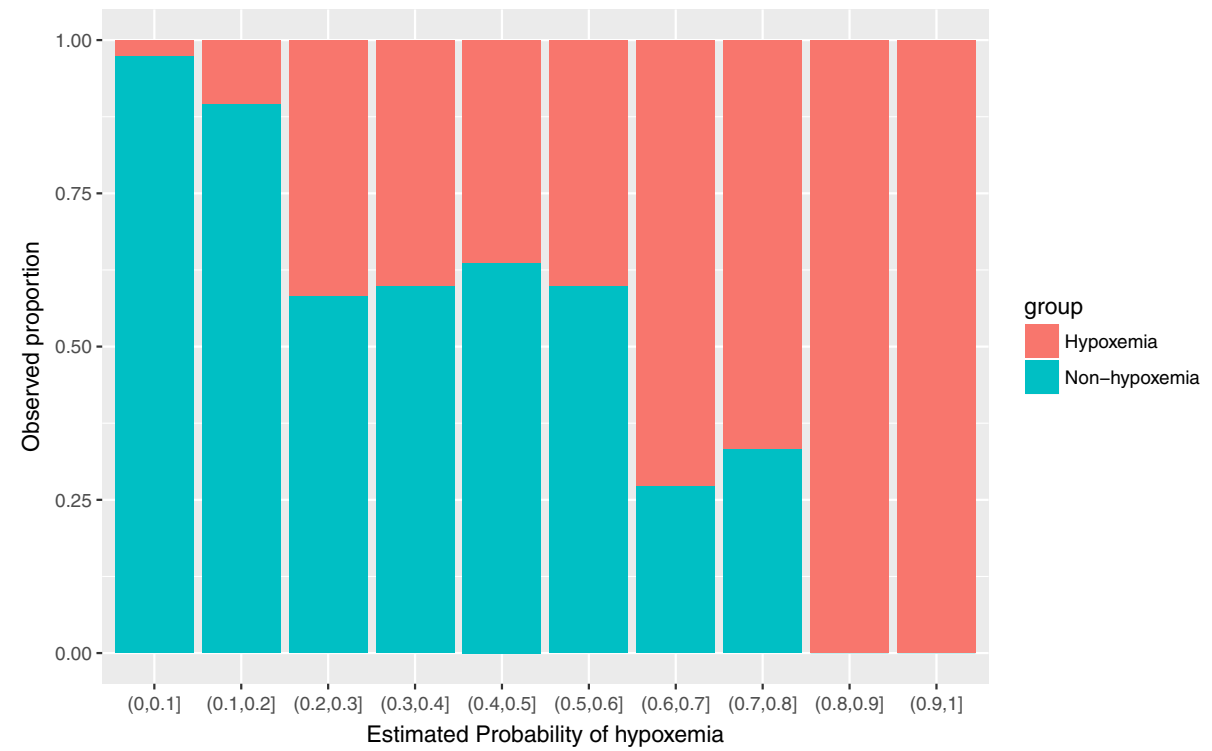

Fig. 3 Bar chart showing the agreement between predicted probability of hypoxemia and observed proportion

points. Points was translated into probability by logit transformation.

\section{Discussion}

The study included patients with AAD who had undergone operation for the repair of the dissection. Risk factors for postoperative hypoxemia were identified via purposeful selection procedure. These factors included age, lactate, preoperative $\mathrm{P} / \mathrm{F}$ ratio, $\mathrm{WBC}, \mathrm{BMI}$ and CRP. Internal validation was performed for the model, which showed good discrimination and calibration. A nomogram was established for clinical utility.

The incidence of hypoxemia was lower than those reported in the literature (20\% vs. 30\%) [4, 14]. Different definitions of postoperative hypoxemia may explain its different incidences. For example, Wang's study defined hypoxemia as $\mathrm{P} / \mathrm{F}$ ratio less than 200 at $24 \mathrm{~h}$ after operation and they reported an incidence of 49.5\% [5]. Several studies have been conducted to explore risk factors for postoperative hypoxemia. Consistent with our study, the study by Liu and colleagues also showed the preoperative $\mathrm{P} / \mathrm{F}$ ratio and $\mathrm{WBC}$ were independently associated with postoperative hypoxemia [4]. It is not surprising that preoperative $\mathrm{P} / \mathrm{F}$ ratio is independently associated with postoperative hypoxemia. Furthermore, $\mathrm{WBC}$ is a biomarker reflecting systemic inflammatory response, and higher responses may contribute to the respiratory dysfunction. There is empirical evidence that inflammatory response is associated with hypoxemia in patients with aortic dissection [15]. Furthermore, we also found CRP was associated with hypoxemia. CRP is a well-known biomarker of inflammatory response, which is more specific and sensitive than WBC $[16,17]$. However, the effect of CRP disappeared after adjusting for covariates. Liu's study also included time from symptom onset and deep hypothermic circulatory arrest time in their regression model. None of the intraoperative variables were associated with hypoxemia in univariate analysis, but the $\mathrm{CPB}$ time was associated with hypoxemia in multivariable regression model. BMI was identified as an independent predictor of postoperative hypoxemia in the study, consistent with the study by Sheng and colleagues [14]. Actually, the association of obesity and hypoxemia is not limited to aortic surgery [18]. In a large cohort of noncardiac surgery, Kendale SM and colleagues found that the odds of experiencing hypoxemia increased significantly with increasing categories of BMI [19]. Similar results were documented in other studies [20,21]. BMI is an important determinant of respiratory function and studies show morbidly obese patients have a typical restrictive pattern with a reduction of forced vital capacity (FVC), forced residual capacity (FRC) and total lung capacity (TLC) with a decreased expiratory reserve volume (ERV) [22-24]. Sex has been found to be related to postoperative hypoxemia in AAD (women were more likely to have hypoxemia than man), which was not replicated in our study. Most probably, the associated was confounded by other factors and the authors failed to adjust for these potential confounding factors [25].

An interesting finding in our study was that serum lactate was associated with postoperative hypoxemia in patients with AAD. Hyperlactatemia is an indicator of tissue ischemia [26]. In cardiac surgery patients, there is evidence that hyperlactatemia is associated with a 


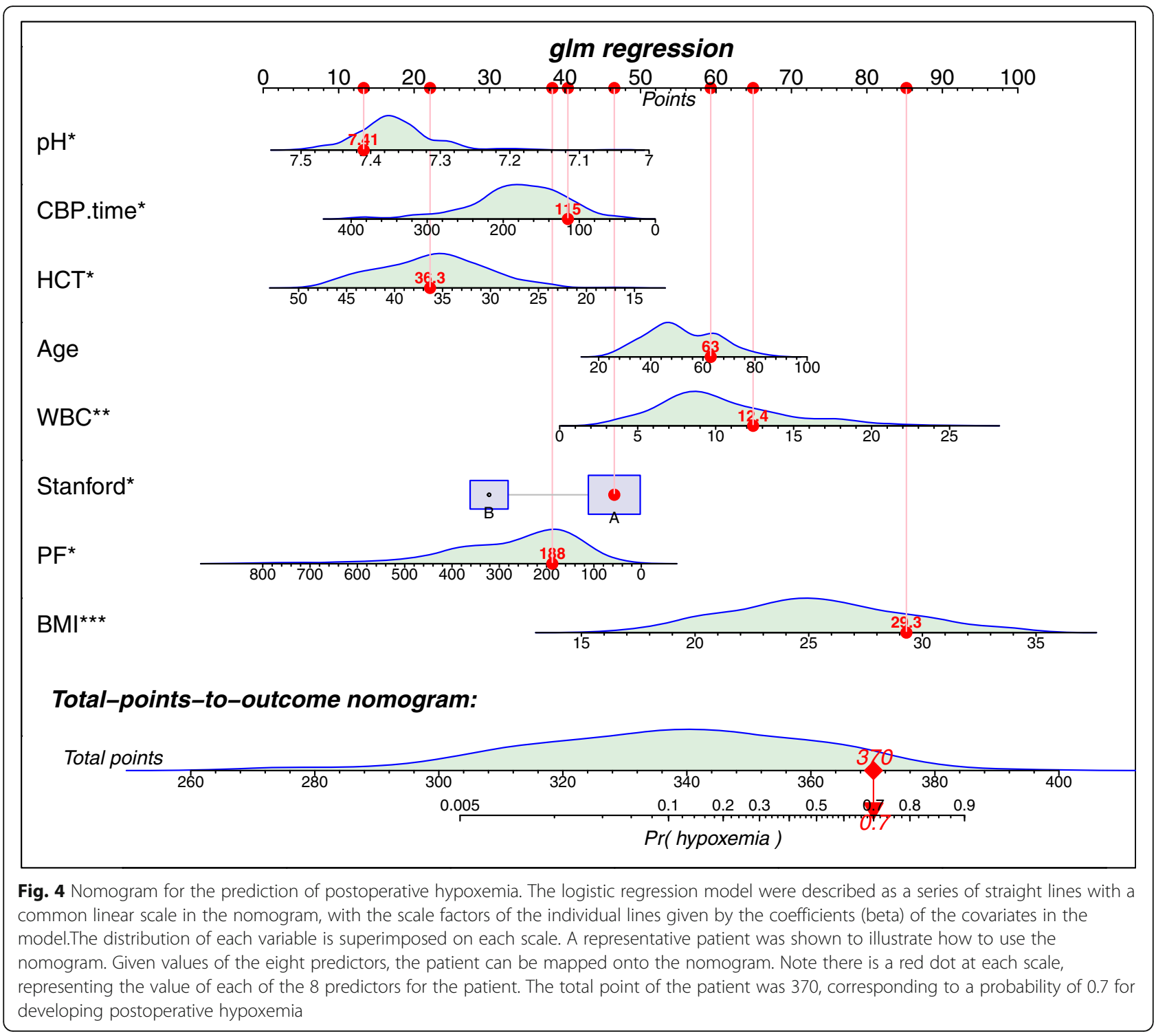

compromised respiratory function and prolonged mechanical ventilation. Also, hyperlactatemia can explain organ dysfunction in our study [27-29]. However, the effect of lactate disappeared in multivariable model, indicating that the effect could be explained by preoperative $\mathrm{P} / \mathrm{F}$ ratio, as lactate was a biomarker of hypoxemia. Similarly, preoperative $\mathrm{P} / \mathrm{F}$ ratio can explain the elevations of biomarkers of acute organ injury such as Scr, bilirubin and ALT. That was why the significant associations in univariate analysis disappeared in the multivariable regression model.

The potential utility of our prediction model is that interventions such as anti-inflammatory agents can be given to patients with high risk of postoperative hypoxemia. Furthermore, the model can be employed to design clinical trials to identify high risk patients who will benefit the most from treatment [30].
There were some limitations in the present study that must be acknowledged. First, the study was retrospective single center study. Although the prediction model was validated in a separate dataset that was not used for model training, its external validity was still unknown. In different cardiac centers, the healthcare process can be quite different and the predictive accuracy of a newly developed model needs to be tested [31, 32]. This is also our future work to perform a multicenter study to examine the external validity of the model. Second, postoperative hypoxemia reported in the study was defined by $\mathrm{P} /$ $F$ ratio less than 200 for consecutive 2 days. While this definition was simple to perform, it suffers from case-mix. Some patients may have intensive respiratory support, and their $\mathrm{P} / \mathrm{F}$ ratio is not comparable to those with spontaneous breathing. Thus, the definition of postoperative hypoxemia needs to be further explored. 
However, in order to make our results comparable to that in the literature [4], we opt to use this simple definition. Third, the retrospective design of the study suffers from its inherent limitation of selection bias. Some patients were excluded because they did not perform operation in our hospital. They might go to other hospital for further evaluation. Thus, the included patients may not well represent the whole target population of $\mathrm{AAD}$, but they represent those who are willing to undergo operation in our hospital. Finally, the sample size of the study was small that only 43 patients had the event of interest. As a rule of thumb, 10-20 events per variable should be used [33]. However, the primary purpose of this rule is to prevent overfitting [34]. We addressed the problem of overfitting by validating the model in a dataset that was not used for model training (e.g. an overfitting model performs poorly in the validation dataset). The results showed that the developed model can predict accurately in the validation dataset with an AUC of 0.869 . Further studies by employing multicenter data are mandatory to endure generalizability of the model.

\section{Conclusion}

In conclusion, the study developed a model for the prediction of postoperative hypoxemia in patients undergoing operation for AAD. Eight variables of age, BMI, WBC, HCT, CPB time, $\mathrm{pH}$, Stanford type and P/ $F$ ratio were included in the model. The model showed good discrimination and calibration in an independent dataset that was not used for model training.

\section{Abbreviations}

AST: Aspartate aminotransferase; BMI: Body mass index; CK: Creatine kinase; CKMB: Creatine kinase isoenzymes; CPB: Cardiopulmonary bypass; CRP: Creactive protein; ICU: Intensive care unit; LDH: Lactate dehydrogenase; LOS: Length of stay; MV: Mechanical ventilation; Scr: Serum creatinine; WBC: White blood cell count

\section{Acknowledgements}

Not Applicable

\section{Funding}

Z.Z. received funding from The public welfare research project of Zhejiang province (LGF18H150005) and Scientific research project of Zhejiang Education Commission (Y201737841);

The funding body had no role in the design of the study and collection, analysis, and interpretation of data and in writing the manuscript.

\section{Availability of data and materials}

Data were not available for confidentiality of the participants.

\section{Authors' contributions}

HG and ZZ conceived of the study; YJ and QJ carried out data entry. LW participated in the data check and interpretation. XQ performed surgical operation and interpreted the results. ZZ performed statistical analysis and drafted the manuscript. All authors read and approved the final manuscript.
Ethics approval and consent to participate

The study was approved by the ethics committee of Sir Run Run Shaw hospital (20180611-7). Informed consent was waived due to retrospective nature of the study.

\section{Consent for publication}

Not applicable.

Competing interests

The authors declare that they have no competing interests.

\section{Publisher's Note}

Springer Nature remains neutral with regard to jurisdictional claims in published maps and institutional affiliations.

\section{Author details}

${ }^{1}$ Department of Respiratory Care, Sir Run Run Shaw Hospital, Zhejiang University School of Medicine, Hangzhou, China. ${ }^{2}$ Department of Cardiovascular Surgery, Sir Run Run Shaw Hospital, Zhejiang University School of Medicine, Hangzhou, China. ${ }^{3}$ Department of Emergency Medicine, Sir Run Run Shaw Hospital, Zhejiang University School of Medicine, No 3, East Qingchun Road, Hangzhou 310016, Zhejiang Province, China.

Received: 9 July 2018 Accepted: 9 October 2018

Published online: 20 October 2018

\section{References}

1. McClure RS, Brogly SB, Lajkosz K, Payne D, Hall SF, Johnson AP. Epidemiology and management of thoracic aortic dissections and thoracic aortic aneurysms in Ontario, Canada: a population-based study. J Thorac Cardiovasc Surg. 2018; 155:2254-64.

2. Nienaber CA, Clough RE. Management of acute aortic dissection. Lancet. 2015;385:800-11.

3. Nakajima T, Kawazoe K, Izumoto H, Kataoka T, Niinuma H, Shirahashi N. Risk factors for hypoxemia after surgery for acute type a aortic dissection. Surg Today Springer-Verlag. 2006;36:680-5.

4. Liu N, Zhang W, Ma W, Shang W, Zheng J, Sun L. Risk factors for hypoxemia following surgical repair of acute type a aortic dissection. Interact Cardiovasc Thorac Surg. 2017;24:251-6.

5. Wang $Y$, Xue $\mathrm{S}$, Zhu H. Risk factors for postoperative hypoxemia in patients undergoing Stanford a aortic dissection surgery. J Cardiothorac Surg BioMed Central. 2013;8:118.

6. Zhang Z, Gayle AA, Wang J, Zhang H, Cardinal-Fernández P. Comparing baseline characteristics between groups: an introduction to the CBCgrps package. Ann Transl Med. 2017;5:484

7. Zhang Z. Univariate description and bivariate statistical inference: the first step delving into data. Ann Transl Med. 2016;4:91.

8. Zhang Z. Model building strategy for logistic regression: purposeful selection. Ann Transl Med. 2016:4:111.

9. Zhang Z. Residuals and regression diagnostics: focusing on logistic regression. Ann Transl Med. 2016:4:195.

10. Zhang Z, Zhang H, Khanal MK. Development of scoring system for risk stratification in clinical medicine: a step-by-step tutorial. Ann Transl Med. 2017:5:436.

11. Harrell FE. rms: Regression Modeling Strategies. 5 ed. 2018. https://cran.rproject.org/web/packages/rms/index.html.

12. Harrell FE. Regression Modeling Strategies. New York: Springer New York; 2001.

13. Zhang Z, Kattan MWK. Drawing nomograms with R: applications to categorical outcome and survival data. Ann Transl Med. 2017;5:190-5.

14. Sheng W, Yang H-Q, Chi Y-F, Niu Z-Z, Lin M-S, Long S. Independent risk factors for hypoxemia after surgery for acute aortic dissection. Saudi Med J. 2015;36:940-6.

15. Duan X-Z, Xu Z-Y, Lu F-L, Han L, Tang Y-F, Tang H, et al. Inflammation is related to preoperative hypoxemia in patients with acute Stanford type a aortic dissection. J Thorac Dis. 2018;10:1628-34.

16. Castelli GP, Pognani C, Cita M, Stuani A, Sgarbi L, Paladini R. Procalcitonin, Creactive protein, white blood cells and SOFA score in ICU: diagnosis and monitoring of sepsis. Minerva Anestesiol. 2006;72:69-80.

17. Zhang Z, Pan L, Deng $H, N i H, X u X$. Prediction of delirium in critically ill patients with elevated C-reactive protein. J Crit Care. 2014;29:88-92. 
18. Kaw RK. Spectrum of postoperative complications in pulmonary hypertension and obesity hypoventilation syndrome. Curr Opin Anaesthesiol. 2017;30:140-5.

19. Kendale SM, Blitz JD. Increasing body mass index and the incidence of intraoperative hypoxemia. J Clin Anesth. 2016;33:97-104.

20. Aizawa K, Sakano Y, Ohki S, Saito T, Konishi H, Misawa Y. Obesity is a risk factor of young onset of acute aortic dissection and postoperative hypoxemia. Kyobu Geka. 2013;66:437-44.

21. Ranucci M, Ballotta A, La Rovere MT, Castelvecchio S, Surgical and Clinical outcome research (SCORE) group. Postoperative hypoxia and length of intensive care unit stay after cardiac surgery: the underweight paradox? PLoS One. 2014;9:e93992.

22. Zhou LN, Wang Q, Gu CJ, Li N, Zhou JP, Sun XW, et al. Sex differences in the effects of obesity on lung volume. Am J Med Sci. 2017;353:224-9.

23. Carey IM, Cook DG, Strachan DP. The effects of adiposity and weight change on forced expiratory volume decline in a longitudinal study of adults. Int J Obes Relat Metab Disord. 1999;23:979-85.

24. Salome CM, King GG, Berend N. Physiology of obesity and effects on lung function. J Appl Physiol. 2010;108:206-11.

25. Maitusong B, Sun H-P, Xielifu D, Mahemuti M, Ma X, Liu F, et al. Sex-related differences between patients with symptomatic acute aortic dissection. Medicine (Baltimore). 2016:95:e3100

26. O'Connor E, Fraser JF. The interpretation of perioperative lactate abnormalities in patients undergoing cardiac surgery. Anaesth Intensive Care. 2012;40:598-603.

27. Zhang Z, Xu X. Lactate clearance is a useful biomarker for the prediction of all-cause mortality in critically ill patients: a systematic review and metaanalysis*. Crit Care Med. 2014;42:2118-25.

28. Zhang Z, Ni H. Normalized lactate load is associated with development of acute kidney injury in patients who underwent cardiopulmonary bypass surgery. PLoS One. 2015;10:e0120466.

29. Lee SM, Kim SE, Kim EB, Jeong HJ, Son YK, An WS. Lactate Clearance and Vasopressor seem to be predictors for mortality in severe Sepsis patients with lactic acidosis supplementing sodium bicarbonate: a retrospective analysis. PLoS One. 2015;10:e0145181.

30. Jin M, Cheng Y, Yang Y, Pan X, Lu J, Cheng W. Protection of xenon against postoperative oxygen impairment in adults undergoing Stanford type-a acute aortic dissection surgery: study protocol for a prospective, randomized controlled clinical trial. Medicine (Baltimore). 2017:96:e7857.

31. Riley RD, Ensor J, Snell KIE, Debray TPA, Altman DG, Moons KGM, et al. External validation of clinical prediction models using big datasets from ehealth records or IPD meta-analysis: opportunities and challenges. BMJ. 2016;353:13140.

32. Bos LD, Schouten LR, Cremer OL, DSY O, Schultz MJ, MARS consortium. External validation of the APPS, a new and simple outcome prediction score in patients with the acute respiratory distress syndrome. Ann Intensive Care. 2016;6:89

33. Ogundimu EO, Altman DG, Collins GS. Adequate sample size for developing prediction models is not simply related to events per variable. J Clin Epidemiol. 2016:76:175-82.

34. Pavlou M, Ambler G, Seaman SR, Guttmann O, Elliott P, King M, et al. How to develop a more accurate risk prediction model when there are few events. BMJ. 2015;351:h3868.

Ready to submit your research? Choose BMC and benefit from:

- fast, convenient online submission

- thorough peer review by experienced researchers in your field

- rapid publication on acceptance

- support for research data, including large and complex data types

- gold Open Access which fosters wider collaboration and increased citations

- maximum visibility for your research: over $100 \mathrm{M}$ website views per year

At BMC, research is always in progress.

Learn more biomedcentral.com/submissions 